\title{
8 LITERACIA EM SAÚdE MENTAL SOBRE ABUSO dE ÁlCOOL EM ADOLESCENTES: DESENVOLVIMENTO DE UM INSTRUMENTO DE MEDIDA
}

\author{
| Amorim Rosa ${ }^{1}$; Luís Loureiro²; Carlos Sequeira ${ }^{3}$
}

\section{RESUMO}

CONTEXTO: As estimativas apontam para que uma em cada cinco crianças e adolescentes venham a ter problemas de saúde mental com expressão antes dos 18 anos de idade e que destes, cerca de metade desenvolva uma perturbação mental com reflexo na idade adulta.

A baixa literacia em saúde mental está associada à ausência de comportamentos de procura de ajuda. Considerando que a promoção da literacia em saúde mental é um pré-requisito para o reconhecimento precoce das perturbações e intervenção atempada, são necessários instrumentos específicos que sustentem a conceção e implementação de programas de educação e sensibilização para a saúde mental ajustados aos contextos e público-alvo.

OBJETIVO: Desenvolver a escala MentaHLiS - AA e analisar as suas propriedades psicométricas.

METODOLOGIA: Participaram no estudo 757 adolescentes entre os 10 e os 18 anos. Para o desenvolvimento do instrumento utilizaram-se metodologias qualitativas (focus group, painéis de peritos). A validade foi verificada através da análise de correspondências múltiplas e da análise fatorial exploratória dos itens, e a fidelidade, através do cálculo do alfa de Cronbach.

RESULTADOS: Da análise resultaram cinco subescalas com índices de fiabilidade aceitáveis, variando entre .718 e .811 , e uma estrutura fatorial consistente com o construto teórico.

CONCLUSÕES: A MentaHLiS - AA é um instrumento fidedigno e valido que pode ser utilizado no rastreio da literacia em saúde mental sobre abuso de álcool e na avaliação do impacto das intervenções de Enfermagem dirigidas à promoção da saúde mental dos adolescentes.

PALAVRAS-CHAVE: Literacia; Saúde mental; Adolescentes; Abuso de substâncias

\section{RESUMEN}

\section{"Alfabetización en salud mental sobre abuso de alcohol en ado- lescentes: Desarrollo de un instrumento de medición"}

CONTEXTO: Las estimaciones indican que uno de cada cinco niños y adolescentes pueden tener problemas de salud mental antes de los 18 años de edad y que de éstos, aproximadamente la mitad desarrollara un trastorno mental en la edad adulta.

La baja alfabetización en salud mental se asocia con la ausencia de comportamientos de búsqueda de ayuda. Considerando que la promoción de la alfabetización en salud mental es un requisito previo para la detección precoz de los problemas e intervención oportuna, son necesarios instrumentos específicos para apoyar el desarrollo e implementación de programas de educación y sensibilización para la salud mental, ajustados a los contextos y público objetivo.

OBJETIVO: Desarrollar la escala MentaHLiS - AA y analizar sus propiedades psicométricas.

METODOLOGÍA: Participaran en el estudio 757 adolescentes entre $10 \mathrm{y}$ 18 años. Para el desarrollo del instrumento se utilizaran métodos cualitativos (grupos focales, grupos de expertos). La validez se verificó mediante análisis de correspondencias múltiples y análisis factorial exploratorio de los ítems, e la confiabilidad a través del alfa de Cronbach.

RESULTADOS: El análisis resultó en cinco subescalas con índices de fiabilidad aceptables $(.718-.811)$, y una estructura factorial consistente con el constructo teórico.

CONCLUSIONES: La MentaHLiS - AA es una herramienta fiable que puede ser utilizada en la detección de la alfabetización en salud mental sobre abuso de alcohol y evaluar el impacto de las intervenciones de enfermería dirigidos a la promoción de la salud mental de los adolescentes.

\section{DESCRIPTORES: Alfabetización; Salud mental; Adolescentes; Abuso de sustancias}

\begin{abstract}
"Mental Health Literacy on alcohol abuse in adolescents: Development of an assessment tool"

BACKGROUND: Recent estimates indicate that around $20 \%$ of children and adolescents under the age of 18 years have mental health problems and that approximately half of them will eventually develop a mental disorder with an impact on adulthood, leading to reduced quality of life and increased use of health care services. Poor mental health literacy is a determining factor for the lack of help-seeking behaviors and the increased risk of recurrence. Mental health literacy is a prerequisite for early recognition and intervention, requiring specific instruments that can serve as a basis for the design and implementation of mental health education and awareness programs adjusted to specific contexts and target groups.

OBJECTIVE: Development and psychometric testing of the MentaHLiS - AA.

METHODS: A total of 757 adolescents aged between 10 and 18 years participated in the study. For the development of the instrument we used qualitative methods (focus group, expert panels). Multiple correspondence analysis and exploratory factor analysis of the items were performed to assess validity and Cronbach's alpha to assess reliability.

RESULTS: Five subscales emerged from the analysis, all with acceptable reliability coefficients (.718 to .811 ), and a factor structure consistent with the theoretical construct.

CONCLUSIONS: The MentaHLiS - AA is a reliable tool that allows the screening of mental health literacy on alcohol abuse and assessing the impact of nursing interventions aimed at the promotion of mental health among adolescents.
\end{abstract}

\section{KEYWORDS: Literacy; Mental health; Adolescents; Substance abuse}

Submetido em 30-09-2016

Aceite em 30-11-2016

1 MSN; Professor Adjunto na Escola Superior de Enfermagem de Coimbra, Rua 5 de Outubro (Polo B), Apartado 7001, 3046-851 Coimbra, Portugal, amorim@esenfc.pt 2 PhD; Professor Adjunto, na Escola Superior de Enfermagem de Coimbra, 3046-851 Coimbra, Portugal, lloureiro@esenfc.pt

3 Doutor em Ciências de Enfermagem; Mestre em Saúde Pública; Enfermeiro especialista em Enfermagem de Saúde Mental e Psiquiátrica; Investigador Principal no Center for Health Technology and Services Research; Coordenador do grupo de investigação "NursID: Innovation \& Development in Nursing"; Professor Coordenador na Escola Superior de Enfermagem do Porto, 4200-072 Porto, Portugal, carlossequeira@esenf.pt

Citação: Rosa, A., Loureiro, L., \& Sequeira, C. (2016). Literacia em saúde mental sobre abuso de álcool em adolescentes: Desenvolvimento de um instrumento de medida. Revista Portuguesa de Enfermagem de Saúde Mental (16), 59-68. 


\section{INTRODUÇÃO}

A adolescência é uma fase crítica para o início das perturbações mentais. As estimativas apontam para que uma em cada cinco crianças e adolescentes venham a ter problemas de saúde mental com expressão antes dos 18 anos de idade (Loureiro et al., 2013), persistindo na idade adulta (Lee et al., 2014) e implicando diminuição da qualidade de vida e aumento do consumo de cuidados de saúde. Contudo, na maioria das situações, os adolescentes não recebem a ajuda adequada ou recebemna com muito atraso (Kelly, Jorm \& Wright, 2007).

$\mathrm{O}$ processo de adolescer pode configurar um período conturbado em torno da busca da identidade, durante o qual as experiências vivenciadas se podem tornar particularmente desestabilizadoras, causando sofrimento psíquico e determinando uma vulnerabilidade acrescida para a exposição ao abuso de substâncias, como é o caso do álcool. A realidade dos conflitos dos adolescentes, como a pressão social, o relacionamento com os pais, a tentativa de extroversão social e a procura de relacionamentos afetivos e sexuais, podem ser alguns dos fatores que indiciam o processo de vulnerabilidade do adolescente em relação ao uso e abuso de álcool (Natividade, Aguirre, Bizarro e Hutz, 2012).

O álcool é a substância psicoativa mais consumida na atualidade sendo o seu uso socialmente aceite e culturalmente justificado. Considerando estes aspetos, podemos dizer que o uso de álcool tem um grande suporte de permissividade social, seja por aspetos sociais históricos ou por campanhas que vão ao encontro da idealização de crianças e adolescentes, numa visão de conquista de público consumidor (Faria, Vendrame, Silva e Pinski, 2011). Em contrapartida, os estudos indicam que até aos 18 anos não deveriam ser consumidas bebidas alcoólicas, porque o fígado não está completamente preparado para metabolizar o álcool. Para além disso, o cérebro encontra-se em desenvolvimento até aos 21 anos de idade, altura em que se conclui a maturação neuronal. Assim sendo, o uso de álcool na adolescência expõe o indivíduo a um maior risco de dependência na idade adulta, pelo que, quanto mais precoce é a fase de experimentação, piores são as consequências e maior é o risco de abuso e dependência de álcool (Wandekoken, Vicente e Siqueira, 2011).

Este facto é particularmente preocupante quando sabemos que as primeiras experiências de consumo ocorrem geralmente no início da adolescência, observandose simultaneamente um consumo excessivo ou abusivo antes dos 18 anos.
Os comportamentos problema decorrentes do consumo excessivo regular ou de episódios pontuais de embriaguez são sobejamente conhecidos. Contudo, durante a adolescência, o consumo de álcool, mesmo em pequenas quantidades, pode ter graves consequências sendo considerado um sério problema de saúde pública (Copeland et al., 2012).

Assim, reconhecendo que o uso precoce de álcool é um problema altamente prevalente entre os adolescentes, a promoção da literacia em saúde mental (LSM) enquanto pré-requisito para o reconhecimento precoce e intervenção atempada (Scott \& Chur-Hansen, 2008), deverá constituir uma preocupação central. Neste sentido têm sido desenvolvidas intervenções no âmbito da educação para a saúde com intuito de prevenir o consumo precoce de álcool e o seu uso inadequado, alertando para os riscos, consequências, efeitos individuais e coletivos. Alguns autores (e.g. Jorm, 2012; Loureiro et al., 2012; Loureiro et al., 2013) sugerem que as intervenções no domínio da promoção da saúde mental dos adolescentes, quando ancoradas no conceito de LSM, tendem a ser eficazes, pois implicam um conhecimento voltado para a ação em proveito da saúde do indivíduo ou dos seus pares. O conceito de LSM foi introduzido em 1997 por Anthony Jorm e colaboradores, sendo definido como os conhecimentos e crenças acerca das perturbações mentais que facilitam o seu reconhecimento, prevenção e/ou gestão. O conceito integra um conjunto de componentes interligados: a) 'capacidade de reconhecer as perturbações de modo a facilitar a procura de ajuda'; b) 'conhecimento da ajuda profissional e tratamentos disponíveis'; c) 'conhecimento da eficácia das estratégias de autoajuda'; d) 'conhecimentos e competências para prestar primeira-ajuda'; e) 'conhecimentos sobre como prevenir as perturbações mentais' (Jorm, 2012; Loureiro et al., 2012).

O conceito releva pela importância do conhecimento mas também pelas crenças associadas que, em conjunto, determinam as atitudes, como por exemplo a resistência em procurar ajuda profissional, perspetivando um conhecimento voltado para a ação (Jorm, 2012). Assim, considerando as limitações substanciais na capacidade atual para avaliar a LSM, é fundamental desenvolver instrumentos que avaliem os seus atributos mais relevantes, servindo de base para a conceção e implementação de programas de educação e sensibilização para a saúde mental e a monitorização o seu efeito. Este estudo tem como objetivo, desenvolver uma escala de avaliação das LSM sobre uso de álcool em adolescentes (MentaHLiS - AA) e avaliar as suas propriedades psicométricas. 


\section{METODOLOGIA}

O estudo foi desenvolvido em quatro fases, utilizando metodologias qualitativas (focus group e painéis de peritos) para o desenvolvimento do instrumento e metodologias quantitativas para avaliação das suas propriedades psicométricas.

Inicialmente foi construída e validada uma vinheta clínica, retratando o caso de um adolescente com critérios para abuso de álcool, segundo o manual Diagnostic and Statistical Manual of Mental Disorders (DSM-IVTR) e a Classificação Internacional de Doenças (CID10). Foram desenvolvidas duas versões da mesma vinheta mudando unicamente o género do adolescente, sendo que cada participante teve acesso apenas a uma das versões.

$\mathrm{Na}$ validação, realizada através do método Delphi, participou um painel de peritos constituído por dois médicos psiquiatras, dois enfermeiros especialistas de Psiquiatria, um psicólogo e dois professores. A vinheta foi avaliada de acordo com três critérios: clareza da redação; adequação à amostra; e adequação ao domínio, sendo pontuada por cada perito com um valor entre 1 e 5 em cada critério. Realizaram-se duas rondas sucessivas até à obtenção de consenso, considerando-se uma pontuação mínima de 28 pontos.

Numa fase seguinte realizaram-se três focus group em escolas secundárias para realizar o pré teste da vinheta e explorar as componentes da LSM sobre abuso de álcool. Participaram 23 adolescentes (16 raparigas e 7 rapazes), com idades compreendidas entre os 12 e os 17 anos, com frequência do $7^{\circ}$ ao $12^{\circ}$ ano. As entrevistas foram sujeitas a análise qualitativa de conteúdo e os resultados permitiram construir os itens da escala.

Numa fase posterior procedeu-se à construção e préteste da escala. Para além de uma secção de caraterização sociodemográfica, a escala integra cinco subescalas, correspondendo a diferentes componentes da LSM: I 'reconhecimento do problema'; II - 'recursos e opções de ajuda'; III - 'crenças e intenções para prestar a primeira ajuda'; IV - 'barreiras e facilitadores na procura de ajuda'; e V - 'crenças sobre comportamentos salutogénicos'.

Os itens das subescalas I,II,III e IV foram definidos em função dos resultados dos focus group. Para além disso, através da revisão da literatura, foi construída uma pole de itens constituintes da subescala V. As questões são avaliadas em escalas tipo Likert de 5 pontos, com exceção da questão sobre o 'reconhecimento do problema' que é de escolha múltipla.
A escala foi submetida a um pré-teste no qual participaram 15 adolescentes. Neste processo procurou-se avaliar a compreensibilidade dos itens, a sua aceitação geral e a previsão do tempo médio de resposta, bem como a identificação de dúvidas e sugestões.

Finalmente, na quarta fase, avaliaram-se as propriedades psicométricas da escala.

Este artigo resulta de um estudo mais amplo de construção de um instrumento de avaliação da LSM, constituído por três escalas (depressão, ansiedade e abuso de álcool), cada uma com cinco subescalas. Destas, três são específicas e com questões direcionadas à situação apresentada na vinheta (I,II e III), enquanto as outras duas (IV e V) avaliam outras dimensões e atributos da LSM.

Assim, para a validação das subescalas IV e V utilizaramse os dados do total da amostra $(n=757)$, enquanto às restantes três responderam apenas 255 indivíduos (159 raparigas e 96 rapazes), com idades compreendidas entre os 10 e os 18 anos $(\mathrm{M}=14.38, \mathrm{DP}=2.20)$, a frequentar 11 escolas públicas pertencentes ao distrito de Coimbra (55.3\% o $3^{\circ}$ ciclo; $32.2 \%$ o secundário; e $12.5 \%$ o $2^{\circ}$ ciclo).

\section{Procedimento}

O estudo foi aprovado pela Direcção-Geral de Educação - Gabinete de Projetos Educativos do Ministério da Educação do Governo Português (n. $\left.{ }^{\circ} 0404700001\right)$ e pela Comissão de Ética da UICISA-E da ESEnfC (P18710-2013). Foi dado o consentimento informado pelos encarregados de educação e pelos estudantes antes da sua inclusão no estudo.

\section{Análise Estatística}

Os dados foram analisados com o software IBM SPSS22. Verificou-se a adesão das distribuições à normalidade das variáveis na população, através do teste de Kolmogorov-Smirnov, utilizando o score total da escala.

Para a subescala I 'reconhecimento do problema', tratando-se de dados nominais (categoriais) com opções de resposta múltipla, foi realizada uma Análise de Correspondências Múltiplas (ACM). Para as restantes subescalas foi verificada a validade de construto através da análise fatorial de componentes principais (ACP) com rotação ortogonal varimax.

A adequação dos dados foi verificada através do teste de esfericidade de Bartlett (BTS) e da medida de KaiserMeyer-Olkin (KMO). 
A escolha das soluções fatoriais finais seguiu os seguintes critérios: Validade convergente - factor loading $\geq$.40; Validade discriminante - diferencial de loading $\geq$ .20 com os restantes fatores; Coeficientes de comunalidade superiores a .40; Concordância entre a estrutura teórica subjacente ao instrumento e a solução encontrada.

A consistência interna foi verificada através do cálculo do coeficiente a de Cronbach.

\section{RESULTADOS}

A versão inicial da escala integrava 71 itens distribuídos em cinco subescalas. A análise fatorial exploratória dos itens e a análise de consistência interna permitiram determinar a sua estrutura interna final, tendo sido eliminados 23 itens, de acordo com os critérios previamente definidos.

Relativamente à subescala I - 'reconhecimento do problema', conforme pode ser observado na Tabela 1, a ACM revelou a existência de dois componentes ou dimensões que explicam na totalidade $40.16 \%$ da variância. Para evitar a análise de categorias pouco representativas consideraram-se unicamente as respostas assinalas com percentagem de concordância superior a $5 \%$ e os itens com valor de discriminação superior à inércia da dimensão. Os valores do alfa de Cronbach mostram um bom ajustamento do modelo: dimensão 1 (.670); dimensão 2 (.369); média com base no autovalor médio (.558).

Tabela 1 - Resultados da Aplicação da Análise de Correspondências Múltiplas aos Itens da Componente Reconhecimento do Abuso de Álcool (N=255)

\begin{tabular}{|l|c|c|}
\hline \multirow{2}{*}{ MentaHLiS - AA } & \multicolumn{2}{|c|}{ Dimensão } \\
\cline { 2 - 3 } & 1 & 2 \\
\hline P20. Anda nervoso e triste & .431 & .007 \\
\hline P11. Tem problemas afetivos & .409 & .060 \\
\hline P17. Tem falta de confiança & .303 & .059 \\
\hline P21. Está revoltado & .279 & .138 \\
\hline P23. Está a reagir a uma perda & .276 & .003 \\
\hline P6. Tem baixa autoestima & .266 & .132 \\
\hline P22. Tem dificuldade em se controlar & .078 & .371 \\
\hline P10. Tem um vício & .180 & .303 \\
\hline P9. Tem falta de responsabilidade & .127 & .237 \\
\hline P2. Tem problemas com a bebida & .169 & .188 \\
\hline Valores próprios (eigenvalues) & 2.52 & 1.50 \\
\hline \% de variância explicada & 25.18 & 14.98 \\
\hline Alfa de Cronbach & .670 & .369 \\
\hline
\end{tabular}

Interpretação Semântica e Nomeação das Dimensões Para a primeira componente são particularmente determinantes as variáveis: "Nervoso e triste", "Problemas afetivos", "Falta de confiança", "Revolta", "Reação a uma perda" e "Baixa autoestima”. Esta dimensão, ao agregar respostas que combinam variáveis semanticamente distintas associadas a atribuições de cariz psicoafectivo e comportamental, configura um 'desconhecimento ou visão difusa da perturbação’ expressa na vinheta.

A segunda componente contempla as variáveis: "Dificuldade em se controlar", "Vício", "Problemas com a bebida " e "Falta de responsabilidade". Nesta dimensão, a associação existente entre os rótulos indica uma perceção do problema como 'doença', sendo esta associada a dificuldades de controlo e falta de responsabilidade.

O "desconhecimento/visão difusa da perturbação" e a "doença" constituem os dois eixos temáticos do espaço em análise.

Nas restantes quatro subescalas, previamente à análise fatorial, verificaram-se os pré-requisitos para a sua utilização. As medidas de adequação amostral KMO das quatro subescalas variaram entre .754 e .836 e os valores do BTS variaram entre 431.05 e 2568.30, (p<.001), o que permitiu prosseguir com a análise.

A matriz de correlações anti-imagem, por sua vez, mostrou que os quadrados das correlações múltiplas (R2), na sua grande maioria, eram superiores a 0,75 (mínimo = .504; máximo $=.889$ ), o que indica um valor ótimo de relação entre as variáveis.

Feita a análise e extraídos os componentes, pretendeuse saber que itens constituiriam cada uma das subescalas e as respetivas dimensões.

Seguindo os critérios previamente definidos foram eliminados os seguintes itens: item 9 (bruxas e ervanárias) da subescala II - porque constituía, por si só, uma dimensão e porque a sua manutenção diminuía significativamente a consistência interna da subescala; itens 5 (sugerir-lhe que fale com um adulto de confiança), 8 (continuar a tratá-la/o como se ele/a não tivesse qualquer tipo de problema) e 9 (sugerir-lhe que beba álcool para esquecer os problemas) da subescala III - o item 5 por ser híbrido, apresentando cargas fatoriais de magnitudes semelhantes (diferenças inferiores a 0,1) em ambos os fatores, e os itens 8 e 9 porque apresentavam correlações negativas muito fortes com a restante subescala e comprometiam a sua consistência interna; os itens 2 (ter consciência do problema e aceitá-lo), 10 (ter a certeza que posso confiar nos profissionais de saúde), 
11 (ter serviços facilmente acessíveis e sem custos), 12 (saber que sozinho poderei não ser capaz de resolver o problema), 13 (ter a certeza que não haverá consequências, como por exemplo ser internado/a) e 14 (conhecer $\mathrm{o} / \mathrm{s}$ profissional/is de saúde) da subescala IV - porque apresentavam correlações com o fator inferiores a .40; loadings semelhante em mais do que um fator; e no caso dos itens 2,11,12 e 14, por apresentarem valores de comunalidade inferiores a .40 .

Apesar dos itens 2 e 8 da subescala II e do item 3 da subescala III violarem, ainda que ligeiramente os critérios definidos (diferença de loadings <.20 e valores de comunalidade $<.40$ ), foram mantidos pela sua importância para o construto teórico e porque a sua exclusão diminuía de forma significativa a consistência interna das subescalas a que pertencem.

No final, as quatro subescalas ficaram constituídas como descrevemos de seguida:

A subescala II 'Recursos e opções de ajuda' apresenta uma estrutura fatorial final com dois fatores que explicam $56.11 \%$ da sua variância total. O primeiro fator designado como 'ajudas formais' integra os itens 3,4,5,6,7 e 8 , explicando $41.72 \%$ da variância. O segundo fator que designámos como 'ajudas informais' Integra os itens 1 e 2 e explica $14.39 \%$ da variância.

A subescala III 'Crenças e intenções para prestar a primeira ajuda' mostrou-se claramente interpretável e ajustada ao construto teórico, numa solução com dois fatores que explicam $59.21 \%$ da variância. O primeiro fator 'apoio social e encaminhamento passivo' explica $41.83 \%$ da variância e é constituído pelos itens 1,2 e 4. O segundo fator 'encaminhamento ativo' integra os itens 3,6,7 e 10, explicando $17.38 \%$ da variância da subescala. A análise dos resultados da subescala IV 'barreiras e facilitadores na procura de ajuda' permitiu verificar que a estrutura fatorial mais interpretável e coerente com o construto teórico seria uma solução com três fatores que, em conjunto, explicam 52.67\% da variância. O primeiro fator 'estigma e preconceito' é composto pelos itens 8,15,16, e 17 e explica $28.09 \%$ da variância. $O$ segundo fator 'determinação e apoio social' integra os itens 1,3,4 e 5 explicando $13.57 \%$ da variância. Finalmente, o terceiro fator que designámos como 'confidencialidade' integra os itens 6,7 e 9, explicando $11.01 \%$ da variância.

Finalmente, a subescala $\mathrm{V}$ 'crenças sobre comportamentos salutogénicos' apresenta uma estrutura interna perfeitamente definida, interpretável e coerente com o construto teórico.
A solução final de três fatores explica $60.35 \%$ da variância total. O primeiro fator 'apoio social positivo' engloba os itens 4,5,6,7 e 8 e explica $22.51 \%$ da variância da subescala. O segundo fator, 'promoção da saúde física e mental' é composto pelos itens 1,2 e 3 e explica $19.67 \%$ da variância. Por fim, o terceiro fator que designámos como 'prevenção de comportamentos de risco' integra os itens 9,10 e 11 e explica $18.17 \%$ da variância total.

$\mathrm{Na}$ Tabela 2 observa-se a configuração final para o agrupamento dos itens em cada uma dos fatores das quatro subescalas. São apresentadas as cargas fatoriais dos itens, os valores de comunalidade, a percentagem de variância explicada e cumulativa, as medidas de KMO e BTS, e ainda, os coeficientes de consistência interna das várias subescalas.

Tabela 2 - Matriz de Saturação dos Itens nos Fatores para Solução Rodada Ortogonal Varimax

\begin{tabular}{|c|c|c|c|c|}
\hline Subescalas & F1 & $\mathrm{F} 2$ & F3 & $h^{2}$ \\
\hline \multicolumn{5}{|l|}{ 'Recursos e opções de ajuda' $(\alpha=.789)$} \\
\hline 1.Amigos de confiança & -.149 & .839 & & .726 \\
\hline 2.Familiares (pais, irmãos) & .329 & .534 & & $(.393)$ \\
\hline 3.Grupos de autoajuda & .552 & .320 & & .407 \\
\hline 4.Psicólogo & .781 & .025 & & .611 \\
\hline 5.Psiquiatra & .783 & -.149 & & .636 \\
\hline 6.Enfermeiro especializado & .806 & .147 & & .671 \\
\hline 7.Médico de família & .729 & .268 & & .604 \\
\hline 8.Professores & .528 & .403 & & .441 \\
\hline Valores próprios (eigenvalues) & 3.338 & 1.151 & & \\
\hline \% Variância explicada & 41.72 & 14.39 & & \\
\hline \multicolumn{5}{|c|}{ 'Crenças e intenções para prestar a primeira ajuda' $(\alpha=.756)$} \\
\hline 1.Dar-lhe apoio e animá-lo/a & .672 & .249 & & .513 \\
\hline $\begin{array}{l}\text { 2.Ouvi-lo/a e tentar compreendê- } \\
\text { lo/a }\end{array}$ & .887 & .006 & & .787 \\
\hline $\begin{array}{l}\text { 3.Encaminhá-lo/a para um profis- } \\
\text { sional de saúde especializado }\end{array}$ & .346 & .450 & & .401 \\
\hline $\begin{array}{l}\text { 4.Acompanhá-lo/a à consulta, não } \\
\text { o/a abandonar }\end{array}$ & .752 & .152 & & .589 \\
\hline $\begin{array}{l}\text { 6.Falar com os familiares para que } \\
\text { estejam mais atentos }\end{array}$ & .400 & .633 & & .561 \\
\hline $\begin{array}{l}\text { 7.Encaminhá-lo/a para um professor } \\
\text { de confiança }\end{array}$ & .097 & .781 & & .620 \\
\hline $\begin{array}{l}\text { 10.Encaminhá-lo/a para o médico } \\
\text { de família }\end{array}$ & .042 & .820 & & .674 \\
\hline Valores próprios (eigenvalues) & 2.929 & 1.216 & & \\
\hline \% Variância explicada & 41.83 & 17.38 & & \\
\hline \multicolumn{5}{|c|}{ 'Barreiras e facilitadores na procura de ajuda' $(\alpha=.718)$} \\
\hline 1.Ter apoio social (pais e amigos) & .172 & .571 & -.109 & $(.368)$ \\
\hline 3.Ter alguém que vá comigo & .031 & .797 & .065 & .641 \\
\hline 4. Ter coragem & .300 & .618 & .145 & .493 \\
\hline $\begin{array}{l}\text { 5.Conhecer alguém que tenha tido } \\
\text { uma boa experiência }(. . .)\end{array}$ & .150 & .567 & .251 & .407 \\
\hline
\end{tabular}




\begin{tabular}{|l|l|l|l|l|}
\hline $\begin{array}{l}\text { 6.Ter a certeza que ninguém vai } \\
\text { saber do meu problema }\end{array}$ & -.149 & .028 & .807 & .674 \\
\hline $\begin{array}{l}\text { 7.Ter a certeza que ninguém me vai } \\
\text { julgar }\end{array}$ & .143 & .179 & .716 & .565 \\
\hline $\begin{array}{l}\text { 8.Não ter medo do julgamento dos } \\
\text { outros }\end{array}$ & .551 & .149 & .240 & $(.383)$ \\
\hline $\begin{array}{l}\text { 9.Ter a certeza que o que disser vai } \\
\text { ser confidencial }\end{array}$ & .334 & .002 & .638 & .519 \\
\hline $\begin{array}{l}\text { 15.Não ter estigma e preconceitos } \\
\text { relativamente a estas doenças }\end{array}$ & .785 & .039 & .110 & .630 \\
\hline 16.Não ter medo das consequências & .741 & .141 & .047 & .571 \\
\hline $\begin{array}{l}\text { 17.Aceitar que procurar ajuda não é } \\
\text { assumir uma fraqueza }\end{array}$ & .667 & .301 & -.095 & .544 \\
\hline Valores próprios (eigenvalues) & 3.090 & 1.493 & 1.211 & \\
\hline \% Variância explicada & 28.09 & 13.57 & 11.01 & \\
\hline 'Crenças sobre comportamentos salutogénicos' $(\alpha=.811)$ & & \\
\hline $\begin{array}{l}\text { 1.Manter hábitos alimentares ad- } \\
\text { equados }\end{array}$ & .138 & .858 & .156 & .779 \\
\hline 2.Manter exercício físico regular & .230 & .822 & .123 & .744 \\
\hline 3.Manter hábitos de sono regulares & .299 & .641 & .112 & .513 \\
\hline 4.Convívio frequente com os amigos & .745 & .180 & -.063 & .591 \\
\hline 5.Ter boa relação com os familiares & .648 & .361 & .116 & .563 \\
\hline 6.Ter sucesso escolar & .661 & .176 & .258 & .535 \\
\hline 7.Ter uma autoestima positiva & .686 & .292 & .158 & .580 \\
\hline $\begin{array}{l}\text { 8.Promover uma boa imagem } \\
\text { corporal }\end{array}$ & .596 & .033 & .184 & .400 \\
\hline $\begin{array}{l}\text { 9.Não consumir álcool, tabaco ou } \\
\text { outras drogas }\end{array}$ & .107 & .134 & .805 & .677 \\
\hline 10.Prevenir o risco de acidentes & .244 & .205 & .678 & .562 \\
\hline 11.Não ter sexo antes da idade & .083 & .048 & .835 & .706 \\
\hline Valores próprios (eigenvalues) & 4.156 & 1.445 & 1.039 & \\
\hline Variância explicada & 22.51 & 19.67 & 18.17 & \\
\hline
\end{tabular}

Tabela 3 - Estatísticas Descritivas e Correlação Média entre os Itens das Subescalas que Compõem a MentaHLiS - AA

\begin{tabular}{|c|c|c|c|c|c|c|}
\hline \multirow{2}{*}{ Subescalas/fatores } & \multirow{2}{*}{$\begin{array}{l}\text { No } \\
\text { Itens }\end{array}$} & \multicolumn{2}{|c|}{ Observado } & \multirow{2}{*}{$\bar{x}$} & \multirow{2}{*}{$\mathrm{s}$} & \multirow{2}{*}{$\begin{array}{l}\text { Correlação } \\
\text { média entre } \\
\text { itens }\end{array}$} \\
\hline & & Min. & Máx. & & & \\
\hline \multicolumn{7}{|c|}{ Recursos e opções de ajuda } \\
\hline 1.Ajudas formais & 5 & 5 & 25 & 19.78 & 3.29 & $.458^{\star}$ \\
\hline 2.Ajudas informais & 3 & 7 & 15 & 12.62 & 1.50 & $.176^{*}$ \\
\hline \multicolumn{7}{|c|}{ Crenças e intenções para prestar a primeira ajuda } \\
\hline $\begin{array}{l}\text { 1.Apoio social e en- } \\
\text { caminhamento passivo }\end{array}$ & 3 & 3 & 15 & 13.66 & 1.61 & $.460^{\star}$ \\
\hline $\begin{array}{l}\text { 2.Encaminhamento } \\
\text { ativo }\end{array}$ & 4 & 4 & 20 & 15.62 & 2.66 & $.366^{\star}$ \\
\hline \multicolumn{7}{|c|}{ Barreiras e facilitadores na procura de ajuda } \\
\hline 1.Estigma e preconceito & 4 & 4 & 20 & 16.64 & 2.45 & $.361^{\star}$ \\
\hline $\begin{array}{l}\text { 2.Determinação e } \\
\text { apoio social }\end{array}$ & 4 & 7 & 20 & 16.94 & 2.16 & $.264^{*}$ \\
\hline 3.Confidencialidade & 3 & 3 & 15 & 10.83 & 2.55 & $.342^{*}$ \\
\hline \multicolumn{7}{|c|}{ Crenças sobre comportamentos salutogénicos } \\
\hline 1.Apoio social positivo & 5 & 8 & 25 & 21.84 & 2.69 & $.386^{\star}$ \\
\hline $\begin{array}{l}\text { 2.Promoção da saúde } \\
\text { física e mental }\end{array}$ & 3 & 3 & 15 & 13.52 & 1.71 & $.518^{\star}$ \\
\hline $\begin{array}{l}\text { 3.Prevenção de com- } \\
\text { portamentos de risco }\end{array}$ & 3 & 3 & 15 & 12.08 & 2.65 & $.471^{\star}$ \\
\hline
\end{tabular}

\section{Análise de Fidelidade}

No estudo de fidelidade procedeu-se à análise da consistência interna através do cálculo do coeficiente alfa de Cronbach. Calculou-se também o coeficiente de correlação corrigido entre o resultado do item e o resultado da subescala de forma a perceber como cada um dos itens se relaciona com o todo. Os itens apresentam correlações moderadas e altas com o score total das subescalas, de um modo geral com valores superiores a .30 , sendo a média de .42 , verificando-se que a exclusão de itens, em todos os 37 casos analisados, não contribuiria significativamente para a elevação do coeficiente a do teste. Excetuam-se duas situações: o item 1 da subescala II e o item 6 da subescala IV. Optou-se pela sua manutenção porque, em ambos os casos, os ganhos de fiabilidade do instrumento seriam pouco significativos. A análise de consistência interna das quatro subescalas resultou em valores de a moderados: subescala II (8 itens), $\alpha=.789$; subescala III ( 7 itens), $\alpha=.756$; subescala IV (11 itens), $\alpha=.718$; e subescala V (11 itens), $\alpha=.811$.

\section{DISCUSSÃO DOS RESULTADOS}

A literatura sugere que o consumo de substâncias tem início sobretudo durante a adolescência, existindo múltiplos fatores relacionados com esta fase desenvolvimental que indiciam um processo de vulnerabilidade do adolescente em relação ao uso e abuso de álcool (Natividade et al., 2012). 
Assim, tendo em conta as limitações atuais para avaliar a LSM, emerge a necessidade de desenvolver instrumentos robustos que avaliem as suas dimensões e atributos mais relevantes.

Este artigo apresenta os resultados do processo de construção e validação de um instrumento fidedigno para avaliar os níveis de LSM sobre abuso de álcool em adolescentes.

Neste estudo usou-se uma vinheta clínica retratando o caso de um adolescente com problemas de abuso de álcool. O recurso a esta estratégia pode ser considerado uma mais-valia porque facilita a avaliação, permitindo a obtenção de respostas mais aproximadas ao que seria o comportamento dos adolescentes em situações reais, ao passo que, quando é usada uma situação hipotética mais simples, as respostas dos participantes não costumam refletir as decisões que eles teriam no seu dia-adia (FeldmanHall et al., 2012).

Os resultados obtidos sugerem-nos alguns comentários:

Das várias componentes da LSM, o reconhecimento do problema emerge como um fator inicial e importante, pois é o primeiro passo para a procura de ajuda de fontes profissionais (Gulliver, Griffiths \& Christensen, 2010).

O reconhecimento é na LSM, entre outras formas, avaliado através da atribuição de rótulos psiquiátricos a diferentes situações do quotidiano que envolvem situações de adoecer mental e/ou sofrimento psicológico (Loureiro, Sequeira, Rosa e Gomes, 2014). Contudo a atribuição de rótulos não pressupõe o diagnóstico em saúde mental, para isso existem profissionais de saúde especializados. Tem antes a intenção mais simples de favorecer ou potenciar a procura de ajuda e o tratamento adequado, quando necessário (Jorm, 2012).

A primeira subescala 'reconhecimento do problema' avalia a capacidade dos adolescentes para reconhecer a perturbação descrita na vinheta através da seleção de rótulos com diferentes níveis de adequação à situação apresentada.

Utilizou-se a ACM, o que nos permitiu agrupar os diferentes rótulos e representá-los em duas dimensões, identificando grupos de indivíduos com perfil semelhante.

Esta estratégia pareceu-nos a mais correta porque a análise das respostas individuais aos rótulos assinalados e às suas combinações permite verificar se o indivíduo é capaz de reconhecer adequadamente o problema (Loureiro et al., 2013).
Por outro lado, a análise fatorial seria uma opção com pouco sentido já que não fornece informação sobre a capacidade para reconhecer adequadamente uma perturbação mental a partir da leitura dos rótulos apresentados (Loureiro, 2015), e porque a análise das relações entre as categorias numa tabela de contingência é mais vantajosa na ACM do que na análise fatorial, sobretudo devido à diferença na informação contida nas dimensões correspondentes (Infantosi, Costa e Almeida, 2014). Considerando que o desenho de programas de intervenção deve partir da análise e avaliação dos défices neste domínio, a criação de scores a partir de loadings dos itens no fator poderá também não fazer sentido, implicando, por vezes, a perda de informação relevante (Loureiro, 2015).

As duas dimensões que emergiram da análise configuram predominantemente um 'desconhecimento ou visão difusa da perturbação', com as respostas que apontam para a perceção do problema como 'doença' a explicarem unicamente $14.98 \%$ da variância da subescala. Este resultado, apesar de preocupante pode ser considerado expectável, justificando os resultados de outros estudos (e.g. Loureiro et al., 2013) que verificaram a existência de dificuldades nos indivíduos para reconhecer e diferenciar o abuso e a dependência de álcool. Por outro lado, o consumo de álcool na sociedade contemporânea é visto com tal permissividade que dificulta o reconhecimento de determinados padrões de consumo como doença (Heckmann e Silveira, 2009). $\mathrm{Na}$ subescala II foram agrupados os itens relacionados com as escolhas dos adolescentes relativamente aos recursos e opções de ajuda para a situação apresentada na vinheta. A análise permitiu identificar duas dimensões antagónicas: uma agrupando os itens relacionados com as opções de ajuda formais ou profissionais; outra integrando as opções de ajuda informais, à semelhança de outros estudos (e.g. Hernan, Philpot, Edmonds \& Reddy, 2010; Rosa, Loureiro e Sousa, 2014) que também identificaram estes dois tipos de opção de ajuda.

Esta variável integra uma das dimensões propostas por Jorm (2012) como componente do conceito de LSM e tem sido sobejamente considerada em outros estudos (e.g. Loureiro et al., 2013; Scott \& Chur-Hansen, 2008). Tal facto releva pela importância atribuída à perceção de que os adolescentes tendem a desvalorizar as ajudas profissionais preferindo apoiar-se nos pares e noutras fontes informais como a família e os professores, numa fase crítica da vida de formação da identidade e socialização, em que as perturbações mentais não tratadas são especialmente negativas, podendo comprometer o desenvolvimento psicossocial e educacional do adolescente (Rosa et al., 2014). 
A subescala III engloba os itens que avaliam de forma global, as competências para prestar a primeira ajuda, integrando-se numa das componentes do conceito de LSM (Jorm, 2012): "first aid skills to support others affected by mental health problems". A subescala aponta para a existência de duas dimensões que traduzem dois tipos de respostas de ajuda já anteriormente utilizadas em outros estudos (e.g. Kelly \& Jorm, 2007): por um lado o encaminhamento ativo com envolvimento de adultos e ajuda profissional; por outro, o apoio social e encaminhamento passivo, traduzido num "apoio distante", sem envolvimento efetivo na ajuda aos pares (Kelly, Jorm \& Rodgers, 2005).

Estas dimensões são particularmente relevantes na avaliação da LSM.

Os estudos mostram que a maioria dos adolescentes sente insegurança e baixa confiança na sua capacidade de ajuda, limitando-se a apoiar e a fazer encaminhamento passivo, com baixa intenção de envolver adultos ou ajudas profissionais (Rosa et al., 2014).

Paradoxalmente estes resultados não encontram eco no estudo de avaliação das propriedades psicométricas do QuALiSMental (Loureiro, 2015) que identificou uma estrutura fatorial complexa, pouco interpretável e com valores de fiabilidade baixos.

A subescala IV compreende três dimensões relacionadas com as barreiras e facilitadores na procura de aju$\mathrm{da}$, constituindo-se como uma das componentes mais importantes do conceito. Vários estudos, utilizando diversos instrumentos e desenhos de investigação, têm analisado os fatores que podem dificultar ou promover a procura de ajuda em situações de doença mental. As dimensões resultantes da análise da subescala (estigma e preconceito; determinação e apoio social; confidencialidade) relacionam-se de forma clara com os resultados desses estudos: confidencialidade, estigma e preconceito (Gulliver et al., 2010; Hernan et al., 2010; Scott \& Chur-Hansen, 2008); determinação e apoio social (Gulliver et al., 2010).

Salienta-se que na versão inicial da escala, os itens destinados à avaliação das barreiras logísticas (e.g. acessibilidade, custos, disponibilidade) não descriminaram de forma a constituir um fator, tendo sido excluídos. Este dado poderá ser parcialmente justificado pelo facto de os participantes no estudo residirem predominantemente em zona urbana e suburbana, com proximidade e disponibilidade de diversos serviços de saúde gratuitos.
Por último a subescala $\mathrm{V}$ que avalia as crenças sobre a importância de manter comportamentos promotores de saúde/saúde mental, integra-se de forma clara na componente "public's knowledge of how to prevent mental disorders" (Jorm, 2012).

Os fatores que emergiram da análise mostram que os adolescentes perspetivam a prevenção, quer pela manutenção e promoção das redes sociais de apoio e, quer ainda pela adoção de comportamentos saudáveis promotores de saúde física e metal e prevenção de comportamentos de risco, tais como evitar a utilização de álcool e de outras drogas, o que tende a associar-se com a intenção de pedir ajuda em saúde mental (Loureiro, 2015).

\section{CONCLUSÕES E IMPLICAÇÕES PARA A PRÁTICA}

Melhorar o conhecimento dos adolescentes sobre as questões de saúde mental pode produzir um impacto positivo na redução do estigma, facilitar a procura de ajuda, e contribuir para o aumento do número de adolescentes com perturbação mental a receber tratamento no futuro.

A MentaHLiS - AA é um instrumento fiável e específico para avaliar a LSM em adolescentes, constituindo uma base que permite ao enfermeiro identificar e reconhecer a existência de problemas, mas também conceber $\mathrm{e}$ implementar programas específicos de educação e sensibilização para a saúde mental em contextos de proximidade que os possam minimizar ou eliminar. Estes programas, pela sua complexidade, exigem instrumentos adequados que permitam monitorizar o seu efeito. A MentaHLiS - AA é, neste sentido, um contributo importante, pois, para além de poder ser aplicada como medida de rastreio da literacia, permite também obter a informação necessária para delinear programas de intervenção inclusivos e adequados aos contextos e público-alvo, capazes de dar resposta a focos de Enfermagem, possibilitando a obtenção de indicadores de resultado positivos, e ainda, avaliar o impacto das intervenções de Enfermagem no domínio da promoção da saúde mental dos adolescentes.

\section{REFERÊNCIAS BIBLIOGRÁFICAS}

Copeland, W. E., Angold, A., Shanahan, L., Dreyfuss, J., Dlamini, I., \& Costello, E. J. (2012). Predicting persistent alcohol problems: A prospective analysis from the Great Smoky Mountain Study. Psychological Medicine, 42(9), 1925-1935. doi: dx.doi.org/10.1017/ S0033291711002790 
Faria, R., Vendrame, A., Silva, R., e Pinski, I. (2011). Propaganda de álcool e associação ao consumo de cerveja por adolescentes. Revista de Saúde Pública, 45(3), 441-447. Acedido em http://www.redalyc.org/ pdf/672/67240191001.pdf

FeldmanHall, O., Mobbs, D., Evans, D., Hiscox, L., Navrady, L., \& Dalgleish, T. (2012). What we say and what we do: The relationship between real and hypothetical moral choices. Cognition, 123(3), 434-441. doi: dx.doi.org/10.1016/j.cognition.2012.02.001

Gulliver, A., Griffiths, K. M., \& Christensen, H. (2010). Perceived barriers and facilitators to mental health help-seeking in young people: A systematic review. Bio Med Central Psychiatry, 10, 113. doi: dx.doi. org/10.1186/1471-244X-10-113

Heckmann, W., e Silveira, C. M. (2009). Dependência do álcool: Aspectos clínicos e diagnósticos. In A. G. Andrade, J. C. Anthony, \& C. M. Silveira, Álcool e suas consequências: Uma abordagem multiconceitual (pp. 67-87). Barueri (SP): Minha Editora. Acedido em http://www.cisa.org.br/UserFiles/File/alcoolesuasconsequencias-pt-cap3.pdf

Hernan, A., Philpot, B., Edmonds, A., \& Reddy, P. (2010). Healthy minds for country youth: Help-seeking for depression among rural adolescents. Australian Journal of Rural Health, 18, 118-124. doi: dx.doi. org/10.1111/j.1440-1584.2010.01136.x

Infantosi, A., Costa, C., e Almeida, R. (2014). Análise de correspondência: Bases teóricas na interpretação de dados categóricos em Ciências da Saúde. Cadernos de Saúde Pública, 30(3), 473-486. doi: dx.doi. org/10.1590/0102-311X00128513

Jorm, A. F. (2012). Mental health literacy: Empowering the community to take action for better mental health. American Psychologist, 67(3), 231-243. doi: dx.doi. org/10.1037/a0025957

Kelly, C., Jorm, A., \& Right A. (2007). Improving mental health literacy as a strategy to facilitate early intervention for mental disorders. British Journal of Psychiatry, 177, 396-401. Acedido em https://www.mja.com.au/ system/files/issues/187_07_011007/kel10278_fm.pdf
Kelly, C., \& Jorm, A. (2007) Adolescents' intentions to offer assistance to friends with depression or conduct disorder: Associations with psychopathology and psychosocial characteristics. Early Intervention in Psychiatry, 1, 150-156. doi: dx.doi.org/10.1111/j.17517893.2007.00009.x

Kelly, M. K., Jorm, A. F., \& Rodgers, B. (2005). Adolescents' responses to peers with depression or conduct disorder. Australian and New Zealand Journal of Psychiatry, 40, 63-66. doi: dx.doi.org/10.1111/j.14401614.2006.01744.x

Lee, F., Heimer, H., Giedd, J., Lein, E., Sestan, N., Weinberger, D., \& Casey, B. (2014). Adolescence mental health, opportunity and obligation. Science, 346, 547549. doi: dx.doi.org/10.1126/science. 1260497

Loureiro, L. (2015). Questionário de avaliação da literacia em saúde mental - QuALiSMental: Estudo das propriedades psicométricas. Revista de Enfermagem Referência, 4(4), 79-88. doi: dx.doi.org/10.12707/RIV14031

Loureiro, L., Mendes, A., Barroso, T., Santos, J., Oliveira, R., \& Ferreira, R. (2012). Literacia em saúde mental de adolescentes e jovens: Conceitos e desafios. Revista de Enfermagem Referência, 3(6), 157-166. Acedido em http://www.scielo.mec.pt/pdf/ref/vserIIIn6/serIIIn6a15.pdf

Loureiro, L. M., Jorm, A. F., Mendes, A. C., Santos, J. C., Ferreira, R. O., e Pedreiro, A. T. (2013). Mental health literacy about depression: A survey about Portuguese youth. Bio Med Central Psychiatry, 13(129), 1-8. doi: dx.doi.org/10.1186/1471-244X-13-129

Loureiro, L., Sequeira, C., Rosa, A., e Gomes, S. (2014). Rótulos psiquiátricos "bem-me-quer, mal-me-quer, muito, pouco e nada...”. Revista Portuguesa de Enfermagem de Saúde Mental (Ed. Esp. 1), 40-46. Acedido em http://www.scielo.mec.pt/pdf/rpesm/nspe1/nspe1a07. pdf

Natividade, J. C., Aguirre, R. C., Bizarro, L., e Hutz, C. S. (2012). Fatores de personalidade como preditores do consumo de álcool por estudantes universitários. Cadernos de Saúde Pública, 28(6), 1091-1100. doi: dx.doi. org/10.1590/S0102-311X2012000600008 
Rosa, A., Loureiro, L., e Sousa, C. (2014). Reconhecimento e procura de ajuda em saúde mental: uma revisão dos estudos realizados em amostras de adolescentes. In L. Loureiro (Coord.), Literacia em saúde mental: Capacitar as pessoas e as comunidades para agir (pp. 77- 91). Coimbra: Unidade de Investigação em Ciências da Saúde-Enfermagem, Escola Superior de Enfermagem de Coimbra.
Scott, L., \& Chur-Hansen, A. (2008). e mental health literacy of rural adolescents: Emo subculture and SMS texting. Australasian Psychiatry, 16(5), 359-362. doi: dx.doi.org/10.1080/10398560802027328

Wandekoken, K. D., Vicente, C. R., e Siqueira, M. M. (2011). Alcoolismo parental e fatores de risco associados. Revista Eletrónica de Saúde Mental. Álcool e Drogas, 7(3). Acedido em: http://pepsic.bvsalud.org/pdf/ smad/v7n3/08.pdf

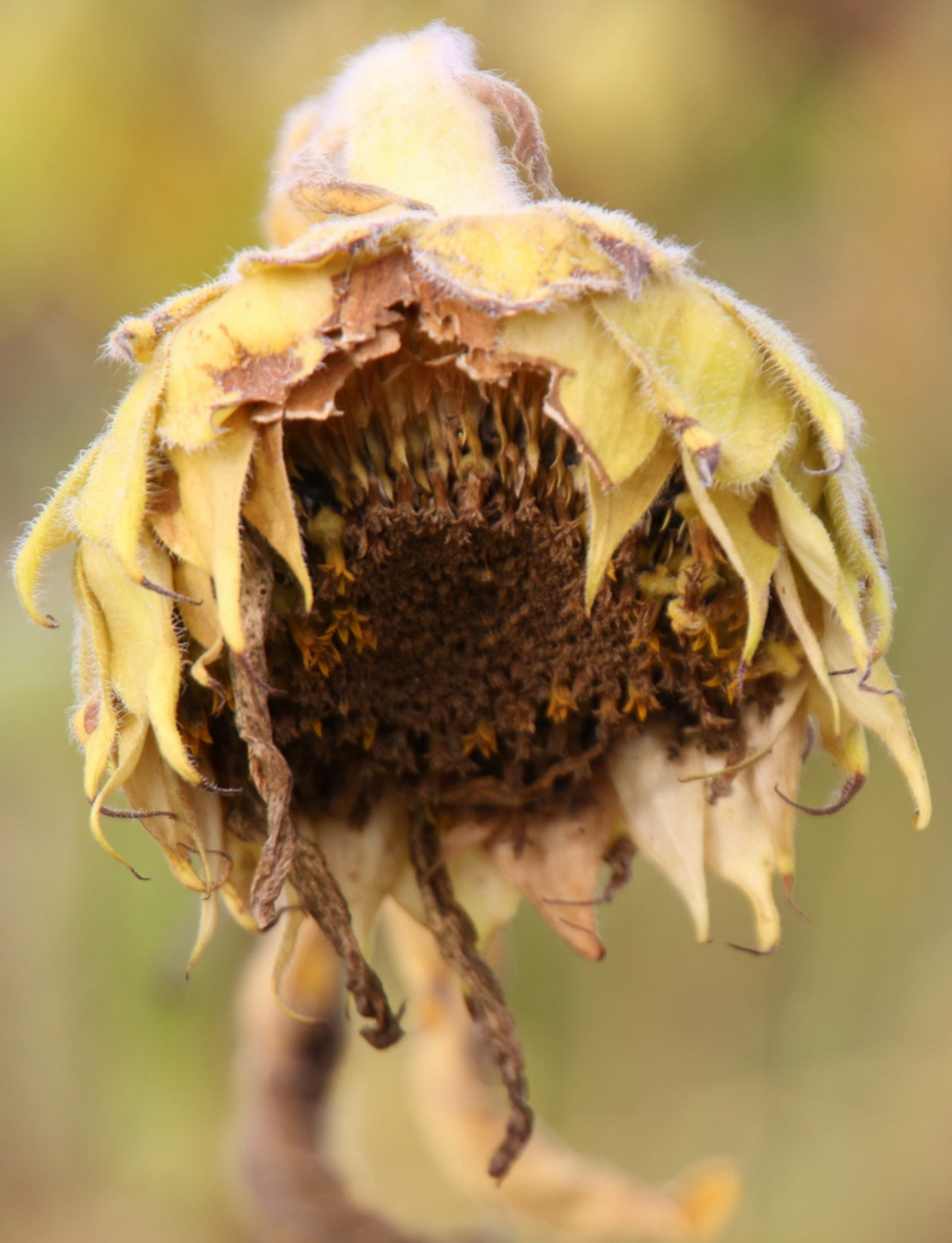

